\title{
Detection of a Proto-planetary Clump in the Habitable Zone of GM Cephei
}

\author{
W. P. Chen, ${ }^{1}$ S. C.-L. $\mathrm{Hu}^{1,2}$ and the YETI Collaboration \\ ${ }^{1}$ Graduate Institute of Astronomy, National Central University, 300 Jhongda Road, \\ Jhongli 32001, Taiwan \\ email: wchen@astro.ncu.edu.tw \\ ${ }^{2}$ Taipei Astronomical Museum, 363 Jihe Rd., Shilin, Taipei 11160, Taiwan
}

\begin{abstract}
GM Cephei is an active T Tauri star in the young open cluster Trumpler 37, showing abrupt UX Orionis type of photometric variability. Its light curves exhibit frequent, sporadic brightening events, each of $<0.5 \mathrm{mag}$ and lasting for days, which must have been originated from unsteady circumstellar accretion. In addition, the star undergoes a brightness drop up to $\sim 1 \mathrm{mag}$ lasting for about a month, during which the star became bluer when fainter. Moreover, the brightness drops seem to have a recurrence timescale of about 300 days. It is proposed that the brightness drop arises from obscuration of the central star by an orbiting dust concentration, exemplifying disk inhomogeneity in transition between grain coagulation and planetesimal formation in a young circumstellar disk. GM Cep was found to show a few percent polarization in the optical wavelengths, and an enhanced level of polarization during the occultation phase.
\end{abstract}

Keywords. occultations; planetary systems: protoplanetary disks; stars: pre-main-sequence; stars: variables: other; stars: individual (GM Cephei)

\section{The Abrupt Young Variable GM Cephei}

Stars are formed out of dense molecular cores, with planets being formed, almost contemporaneously, in the young circumstellar disks. The grain growth process initiated in the parental molecular cloud continues to produce progressively larger solid bodies. Details are still lacking in how grain coagulation proceeds to eventual planet formation in a turbulent disk. Competing theories include gravitational instability (Safronov 1972, Goldreich \& Ward 1973, Johansen et al. 2007) and planetesimal accretion (Weidenschilling 2000). In any case, density inhomogeneities in the young stellar disk should mark the critical first step in the process. The clearing time scale of optically thick disks has been estimated to be less than 10 Myr (Mamajek, Briceño et al. 2007, Hillenbrand 2008). This epoch corresponds to pre-main sequence (PMS) stellar evolution from disk-bearing classical T Tauri stars to weak-lined T Tauri stars with no optically thick disks.

PMS objects are known to vary their brightness and colors due to accretion of or obscuration by circumstellar material. Herbst et al. (1994) attributed young stellar variability to: (i) rotational modulation of cool star spots. (ii) unsteady accretion onto a hot spot on the stellar surface. These stars are called EX Lupi type variables or EXors. (iii) obscuration by circumstellar dust clumps. These are UX Orionis type variables or UXors. About a dozen UXors are known, with some showing variability with periods ranging from $8.2 \mathrm{~d}$ (Bouvier et al. 2003) to $11.2 \mathrm{yr}$ (Grinin et al. 1998).

GM Cephei is an active T Tauri star in the young $(<4$ Myr, Marschall, Karshner, \& Comins 1990; Patel et al. 1995; Sicilia-Aguilar tal 2005) cluster Trumpler 37. At a distance of $870 \mathrm{pc}$ (Contreras et al. 2002), the cluster is associated with the prominent 


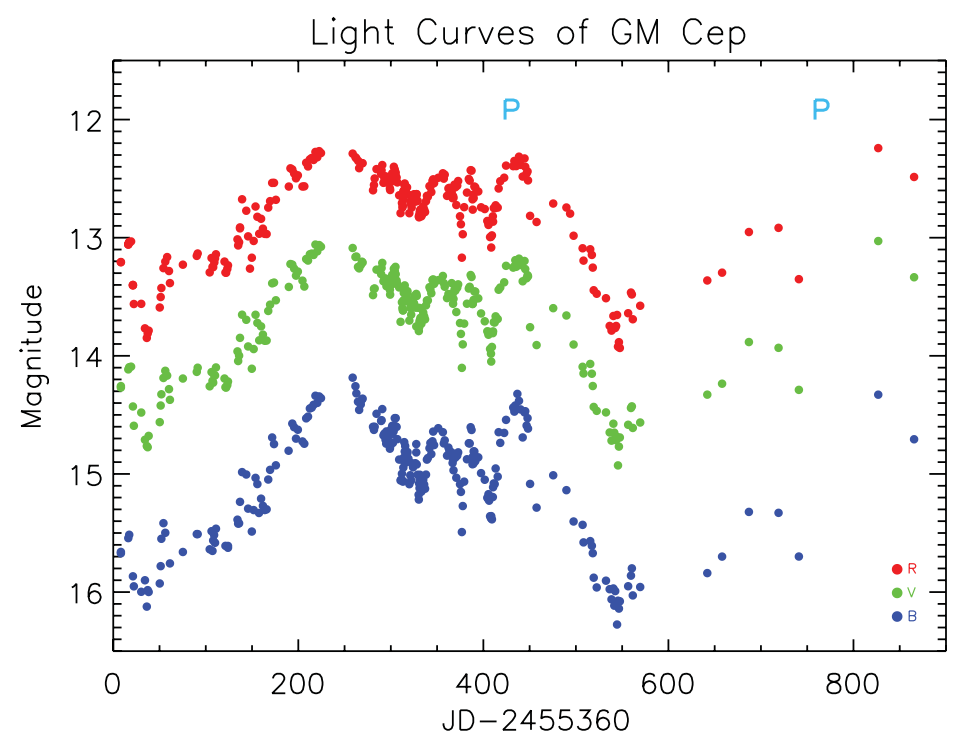

Figure 1. Light curves of GM Cep since 12 June 2010 (JD=2455360).

H II region IC 1396, bright-rimmed clouds, and is populated with young stars. GM Cep is known to be an abrupt variable, but interpretations of its variability have been controversial. Sicilia-Aguilar et al. (2008) suggested GM Cep to be an EXor. But Xiao, Kroll, \& Henden (2010) concluded that the variability in the light curve is dominated by dips superposed on quiescent states. That GM Cep should be a UXor was later supported by studies of Semkov \& Peneva (2012) and Chen et al. (2012).

\section{Occultation by a Circumstellar Clump}

Chen et al. (2012) presented light curves and color variation of GM Cep up to 2011, as part of the Young Exoplanet Transit Initiative (YETI) collaboration, a global network of small telescopes to search for exoplanet transit events in young star clusters (Neuäuser et al. 2011). GM Cep was found to show sporadic flare events, each with brightening of $<0.5 \mathrm{mag}$ lasting for days. Such abrupt light curves are seen among some Herbig Ae/Be stars, with "cyclic but not exactly periodic" (Herbst \& Shevchenko 1999) behavior. The flares detected in GM Cep were associated with a blue color, so should originate from an increased accretion activity. In addition, GM Cep also underwent a brightness drop of $\sim 1$ mag with the duration of about a month, when the star became bluer when fainter. Such brightness drops seem to have a recurrence time scale of 310-320 d. Interestingly, between consecutive drops, the star brightened then dimmed gradually by about $1 \mathrm{mag}$, similar to what is seen in the light curves of eclipsing binaries or exoplanet transits, and became bluer when brighter. Fig. 1 shows the light curves in $B, V$, and $R$ bands up to 2012. The dip and brightening showed up clearly from end of 2011 to mid-2012, confirming the cyclic nature of the drop event, with a repetitive time of $\sim 500 \mathrm{~d}$.

It was proposed that the brightness drop was caused by obscuration of the central star by an orbiting dust concentration (Chen et al. 2012). What is seen in GM Cep therefore is a manifestation of density inhomogeneity of dust in a young stellar disk. From the depth, duration, and period of the drop event, Chen et al. (2012) derived the column density of $N_{d}=2.0 \times 10^{9} \mathrm{~cm}^{-2}$ (for dust only), a size extent of $0.8 \mathrm{AU}$, and an orbital radius of $1.2 \mathrm{AU}$ for the obscuring "clump". With some assumptions, a total dust mass of $2.3 \times 10^{21} \mathrm{~g}$ was derived. The density contrast is not necessarily high relative to the 
neighboring disk material, so the "clump" may be a mere dust concentration in a warped or spiral-armed disk (perhaps excited by a companion star). Because the occultation has been repeatedly seen for at least a few orbits, the concentration must be dynamically stable, and may be a signpost of the transition phase from grain growth to the onset of planetesimal formation. Though rare, GM Cep is not an isolated case among young stars (Hillenbrand et al. 2013).

\section{POLARIZATION OBSERVATIONS}

Optical polarization of GM Cep has been measured by TRIPOL (Triple-Range Imaging Polarimeter), an imaging polarimeter taking data simultaneously at the $g^{\prime}, r^{\prime}$, and $i^{\prime}$ bands. TRIPOL uses dichroic mirrors to split the beam from the telescope into three SBIG ST-9XE camera detectors, and with a wire-grid polarizer provides an efficient, flexible, compact, and economic solution for polarization measurements with small telescopes. The ST-9XE camera has $512 \times 512$ pixels, with each pixel of $20 \mu \mathrm{m}$ on a side. Each data set consists of sequential exposures at 0, 45, 22.5 and $67.5 \mathrm{deg}$, from which the Stokes parameters, and accordingly the polarization vector of a star is derived. Polarized and unpolarized standard stars are observed to calibrate the instrument polarization.

GM Cep was observed on 17 and 18 August 2011 using TRIPOL mounted on the Lulin One-meter Telescope. The star was found to be moderately polarized, with $P_{g}=$ $5.6 \pm 0.4 \%, \theta_{g}=65 \pm 1$ at $g, P_{r}=5.0 \pm 0.2 \%, \theta_{r}=70 \pm 1$ at $r$, and $P_{i}=4.0 \pm 0.2 \%$, $\theta_{i}=69 \pm 1$ at $i$, where $\theta$ is the position angle in degree eastwards from north. The star was observed again on 17-19 July 2012 when the star was expected to brighten to maximum. Preliminary analysis indicates $P_{g}=7.9 \pm 0.3 \%, \theta_{g}=66 \pm 1, P_{r}=6.8 \pm 0.1 \%, \theta_{r}=69 \pm 1$, and $P_{i}=5.8 \pm 0.1 \%, \theta_{i}=66 \pm 1$, suggestive of a slight increase in polarization level at all wavelengths with little change in position angle. Both sets were measured during the back scattering phase, lending tentative support to the dust clump scenario. Polarization observations are being planned for the next occultation in early 2013.

\section{References}

Bouvier, J., et al. 2003, A\&A, 409, 169

Briceño, C., et al. 2007, ApJ, 661, 1119

Chen, W. P., et al. 2012, ApJ, 751, 118

Contreras, M. E., et al. 2002, AJ, 124, 1585

Goldreich, P. \& Ward, W. 1973, ApJ, 183, 1051

Grinin, V. P., Rostopchina, A. N., \& Shakhovskoi, D. N. 1998, Ast. Lett., 24, 802

Herbst, W., Herbst, D. K., Grossman, E. J., \& Weinstein, D. 1994, AJ, 108, 1906

Herbst, W. \& Shevchenko, V. S. 1999, AJ, 118, 1043

Hillenbrand, L. A. 2008, Phys. Scr., 130, 4024

Hillenbrand, L. A., et al. 2013, AJ, 145, article id. 59

Johansen, Anders, et al. 2007, Nature, 448, 1022

Mamajek, E. E. et al. 2004, ApJ, 612, 496

Marschall, L. A., Karshner, G. B., \& Comins, N. F. 1990, AJ, 99, 1536

Neuäuser, et al. 2011, AN, 332, 527

Patel, N., et al. 1995, ApJ, 447, 721

Safronov, V. S. 1972, in Evolution of the protoplanetary cloud and formation of the earth and planets, Jerusalem: Keter Publishing House

Semkov, E. H. \& Peneva, S. P. 2012, Ap\&SSS, 338, 95

Sicilia-Aguilar, A., et al. 2005, AJ, 130, 188

Sicilia-Aguilar, A., et al. 2008, ApJ, 673, 382

Weidenschilling, S. 2000, Sp. Sci. Rev., 92, 295

Xiao, L., Kroll, P., \& Henden, A. 2010, A, 139, 1527 